University of Nebraska - Lincoln

DigitalCommons@University of Nebraska - Lincoln

May 2008

\title{
Phase-field modeling of the formation of lamellar nanostructures in diblock copolymer thin films under inplanar electric fields
}

Xiang-Fa Wu

Department of Engineering Mechanics, University of Nebraska-Lincoln, xfwu@unlserve.unl.edu

Yuris A. Dzenis

Department of Engineering Mechanics, University of Nebraska-Lincoln, ydzenis@unl.edu

Follow this and additional works at: https://digitalcommons.unl.edu/engineeringmechanicsfacpub

Part of the Mechanical Engineering Commons

Wu, Xiang-Fa and Dzenis, Yuris A., "Phase-field modeling of the formation of lamellar nanostructures in diblock copolymer thin films under inplanar electric fields" (2008). Faculty Publications from the Department of Engineering Mechanics. 10.

https://digitalcommons.unl.edu/engineeringmechanicsfacpub/10

This Article is brought to you for free and open access by the Mechanical \& Materials Engineering, Department of at DigitalCommons@University of Nebraska - Lincoln. It has been accepted for inclusion in Faculty Publications from the Department of Engineering Mechanics by an authorized administrator of DigitalCommons@University of Nebraska - Lincoln. 


\title{
Phase-field modeling of the formation of lamellar nanostructures in diblock copolymer thin films under inplanar electric fields
}

\author{
Xiang-Fa $\mathrm{Wu}^{*}$ and Yuris A. Dzenis \\ Department of Engineering Mechanics, Nebraska Center for Materials and Nanoscience, University of Nebraska-Lincoln, \\ Lincoln, Nebraska 68588-0526, USA \\ (Received 27 June 2007; revised manuscript received 4 February 2008; published 26 March 2008)
}

\begin{abstract}
Recent experiments show that external inplanar electric field can be employed to guide the molecular self-assembly in diblock copolymer (BCP) thin films to form lamellar nanostructures with potential applications in nanotechnology. We study this self-assembly process through a detailed coarse-grained phaseseparation modeling. During the process, the free energy of the BCP films is modeled as the Ginzburg-Landau free energy with nonlocal interaction and electrostatic coupling. The resulting Cahn-Hilliard $(\mathrm{CH})$ equation is solved using an efficient semi-implicit Fourier-spectral algorithm. Numerical results show that the morphology of order parameter formed in either symmetric or asymmetric BCP thin films is strongly influenced by the electric field. For symmetrical BCPs, highly ordered lamellar nanostructures evolved along the direction of the electric field. Phase nucleation and dislocation climbing in the BCP films predicted by the numerical simulation are in a good agreement with those observed in recent BCP electronanolithography. For asymmetrical BCPs, numerical simulation shows that nanodots are guided to align to the electric field. Furthermore, in the case of high electric field, nanodots formed in asymmetrical BCPs may further convert into highly ordered lamellar nanostructures (sphere-to-cylinder transition) parallel to the electric field. Effects of the magnitude of electric field, BCP asymmetry, and molecular interaction of BCPs on the self-assembly process are examined in detail using the numerical scheme developed in this study. The present study can be used for the prediction of the formation of nanostructures in BCP thin films and the quality control of BCP-based nanomanufacturing through optimizing the external electric fields.
\end{abstract}

DOI: 10.1103/PhysRevE.77.031807

PACS number(s): 83.80.Uv, 81.16.Rf, 82.35.Jk, 89.75.Kd

\section{INTRODUCTION}

Thin films of diblock copolymers (BCPs) bear the natural tendency to form periodical morphology in nanoscale that provides an efficient technique to produce nanostructured materials and nanodevices [1-7]. With the aid of proper external fields, molecular self-assembly in BCP thin films can be guided to form long-range ordered nanostructures. This has evoked increasing interests due to its promising applications in nanotechnology-based products such as high-density random-access memory devices, increased capacitance gate devices, acoustic transducer arrays, photonic imaging arrays, etc. [8-13]. To date, quite a few techniques have been developed for the fabrication of long-range ordered nanostructures in BCP thin films (e.g., chemically patterned substrates [14-20], topological templates [13,21-25], electric fields [26-34], elastic fields [35-37], shear flows [38-45], etc.). One may find that external electric field may be among the most efficient means, which has attracted substantial attention since the original work achieved in Russell's and Jaeger's groups [26-28]. Recent endeavor has also been dedicated to understanding the alignment mechanisms and the molecular self-assembly process in BCP thin films in electric fields. For instance, it was reported recently that when subjected to an inplanar electric field of sufficient magnitude, sphere-to-cylinder domain transition happened in polystyrene- $b$-poly (methyl methacrylate) (PS- $b$-PMMA) thin films [32]. In such process, in attempting to reduce the

\footnotetext{
*xfwu@unlserve.unl.edu
}

global free energy of the PS- $b$-PMMA thin films, the spherical microdomains that nucleated in the thin films were gradually deformed into ellipsoids and consequently interlinked to form cylindrical lamellar microdomains along the direction of the applied electric field.

Accordingly, efficient computational modeling of phaseseparation process provides a convenient method to understand and predict the nanostructure formation process in BCP thin films. Two basic numerical schemes have been employed extensively. The first is based on the dynamic selfconsistent field (SCF) simulation, and the second is the cell dynamics simulation (CDS). In principle, CDS can be implemented to capture the entire evolution process of the molecular self-assembly, which is also more time-efficient compared to SCF. For SCF, the large demand of the computational time is mainly due to the heavy calculation of the path integrals for the chain conformation. In addition, several research groups have examined the sphere-to-cylinder transition process in BCP thin films by using either SCF [46-52] or CDS [32,53-55]. Predictions based on the computational models were very close to those observed in experiments. This indicates the power of computational modeling in nanodevice design and prediction of nanostructure formation. Due to the rapid increase of computational cost with increasing problem scale, explicit CDS can be only fulfilled on a computational cell with intermediate number of grids. Large scale CDS modeling, especially in the case of general three-dimensional modeling, has to resort to the computational capacity of supercomputers. Also, the time step used in CDS has to be very small in order to satisfy the stability requirement of the explicit algorithms. Thus, further research is still desired in 
enhancing the computational efficiency and numerical reliability in modeling the molecular self-assembly in BCPs.

Among these efforts, in this study, we present the results from a detailed numerical study of the molecular selfassembly in BCP thin films subjected to an inplanar electric field. During the process, the free energy of the BCP thin films is modeled as the Ginzburg-Landau free energy with nonlocal interaction and electrostatic coupling [56-60]. The resulting Cahn-Hilliard $(\mathrm{CH})$ equation is solved using an efficient semi-implicit Fourier-spectral algorithm $[61,62]$. This numerical scheme is unconditionally stable; therefore the time steps used in iterations can be selected much larger than those used in the conventional coarse-grained phaseseparation modeling (e.g., CDS). This numerical scheme has been examined successfully in capturing the evolution of phase domains in BCP thin films on chemically patterned substrates [63]. It shows great numerical stability and computational efficiency in time iteration. Thus, in this study, by using this numerical scheme, we are going to further examine the effects of BCP asymmetry, molecular interaction, and magnitude of the applied electric field on the molecular selfassembly process in BCP thin films.

The remainder of this paper is organized as follows. The derivation of the governing $\mathrm{CH}$ equation of the $\mathrm{BCP}$ thin films and relevant numerical algorithm are presented in Sec. II. Numerical results for the evolution of lamellar nanostructures in symmetric BCP films and the sphere-to-cylinder domain transition in asymmetric BCP films are detailed in Sec. III. Conclusions and potential applications of the present study are addressed in Sec. IV.

\section{MODEL FORMULATION AND NUMERICAL PROCEDURE}

Molecular self-assembly in BCPs can be described by using the Landau-Ginzburg phenomenological model of phase separation. Thus, the time-dependent free energy functional for a general BCP confined on a flat substrate free of external field can be expressed [56-60] as

$$
\begin{aligned}
\frac{F}{k_{B} T}= & \int\left(f[\varphi(\vec{r}, t)]+\frac{K}{2}|\nabla \varphi(\vec{r}, t)|^{2}\right) d \vec{r}+\frac{A_{0}}{2} \int G\left(\vec{r}, \vec{r}^{\prime}, t\right) \\
& \times\left[\varphi(\vec{r}, t)-\varphi_{0}\right]\left[\varphi\left(\vec{r}^{\prime}, t\right)-\varphi_{0}\right] d \vec{r} d \vec{r}^{\prime}
\end{aligned}
$$

where the interface energy between the BCP and the substrate is assumed constant and therefore ignored since it does not affect the phase separation process in the present study. $k_{\mathrm{B}}$ is the Boltzmann constant; $T$ is the absolute temperature; $K$ is a constant to delineate the phase boundary energy; $\vec{r}$ is the local position vector; $f[\varphi(\vec{r}, t)]$ is the local coarse-grained free energy density of mixing that takes the Landau form such that $f[\varphi(\vec{r}, t)]=-\varphi(\vec{r}, t)^{2} / 2+\varphi(\vec{r}, t)^{4} / 4 . \varphi(\vec{r}, t)$ is the local concentration difference between two different BCP monomers that are assumed as $A$ and $B$ such that $\varphi(\vec{r}, t)$ $=2 \phi(\vec{r}, t)-1$, (i.e., the order parameter), where $\phi(\vec{r}, t)$ is the local volume fraction of the $A$ monomer in the $A-B$ BCP. $G\left(\vec{r}, \vec{r}^{\prime}, t\right)$ is the Green's function for the Laplace equation such that $\nabla^{2} G=-\delta(\vec{r}-\vec{r}) . \varphi_{0}$ is the mean value of the order parameter averaging spatially. For $\varphi_{0}=0$, the above free energy functional recovers that of a symmetric BCP.
In this study, the BCP films are assumed very thin, i.e., monolayer as used in BCP nanolithography recently $[6-11,16,18,20]$. As a result, the volumetric integrals above can be reduced to those with respect to the substrate surface. In reality, after a quench from the high-temperature disordered melting state to a temperature below the microphase segregation temperature, stable phases will form through phase separation in order to reduce the global free energy of the BCP system. This process can be modeled by the $\mathrm{CH}$ equation or model B in the Hoherberg-Halperin notation [64] such that

$$
\frac{\partial \varphi}{\partial t}=\nabla\left(M \nabla \frac{\delta F(\varphi, t)}{\delta \varphi}\right),
$$

where $M$ is the mobility to be considered as constant for convenience. Substituting Eq. (1) into Eq. (2) and then rescaling it with $M$ yields the dimensionless $\mathrm{CH}$ equation on the substrate surface

$$
\frac{\partial \varphi}{\partial t}=\nabla^{2}\left(-\varphi+\varphi^{3}-\nabla^{2} \varphi\right)+\alpha\left(\varphi-\varphi_{0}\right),
$$

where $\alpha$ is a compound dimensionless phenomenological parameter that characterizes the nonlocal $A-B$ potential $A_{0}$, the molecular chain length, and the mobility $M$. When an external electric field is adopted along the $x$ axis in the given BCP system, an additional term contributed by the electric field is added into Eq. (3),

$$
\frac{\partial \varphi}{\partial t}=\nabla^{2}\left(-\varphi+\varphi^{3}-\nabla^{2} \varphi\right)+\alpha\left(\varphi-\varphi_{0}\right)+\beta \frac{\partial^{2} \varphi}{\partial x^{2}} .
$$

Here $\beta=\kappa \varepsilon_{0}\left(\varepsilon_{A}-\varepsilon_{B}\right)^{2} E_{0}^{2} /(k T), \varepsilon_{0}$ is the vacuum permittivity, $\varepsilon_{A}$ and $\varepsilon_{B}$ are the dielectric constants of the pure $A$ and $B$ homopolymer phases of the BCP film, respectively, and $\kappa$ is a compound dimensionless parameter. The derivation of the electric contribution is based on linear perturbation theory. Similar derivation is also available in the literature $[53-55,65,66]$. The modified $\mathrm{CH}$ equation (4) can be solved conveniently by means of explicit finite difference methods within a representative computational cell under periodic boundary conditions well discussed in the literature. Hereafter, we adopt the efficient semi-implicit Fourier-spectral method [61-63] to solve Eq. (4). To do so, by performing double Fourier transform with respect to the spatial variables $x$ and $y$ at two sides of Eq. (4), we obtain the $\mathrm{CH}$ equation (4) in the time-Fourier domain

$$
\frac{\partial \bar{\varphi}}{\partial t}=k^{2}\left(\bar{\varphi}-\overline{\varphi^{3}}-k^{2} \bar{\varphi}\right)-\alpha\left(\bar{\varphi}-\bar{\varphi}_{0}\right)-\beta k_{x}^{2} \bar{\varphi},
$$

where $k=\sqrt{k_{x}^{2}+k_{y}^{2}}$ with $k_{x}$ and $k_{y}$ as the variables in the Fourier domain, and variables with upper bars denote the double Fourier transforms of the corresponding variables in the physical space. For instance, $\bar{\varphi}(k, t)$ is the double Fourier transform of $\varphi(x, y, t)$, i.e., $\bar{\varphi}(k, t)$ $=\int_{-\infty}^{+\infty} \int_{-\infty}^{+\infty} \varphi(x, y, t) \exp \left[-i\left(k_{x} x+k_{y} y\right)\right] d x d y$. In order to discretize Eq. (5) in the time domain for the use of the semiimplicit numerical scheme, we deal with the terms in Eq. (5) separately. The key idea is that treating the linear terms im- 
plicitly can reduce the stability constraints; while treating the nonlinear term explicitly can avoid directly solving the nonlinear equation at each time step during the numerical iteration. Consequently, we obtain the final form of the discretized equation

$$
\begin{aligned}
\left(\bar{\varphi}^{n+1}-\bar{\varphi}^{n}\right) /(\Delta t)= & k^{2} \bar{\varphi}^{n+1}-k^{2} \bar{\varphi}^{3 n}-k^{4} \bar{\varphi}^{n+1}-\alpha \bar{\varphi}^{n+1}+\alpha \bar{\varphi}_{0} \\
& -\beta k_{x}^{2} \bar{\varphi}^{n+1},
\end{aligned}
$$

which leads to the iteration format as

$$
\bar{\varphi}^{n+1}=\frac{\bar{\varphi}^{n}+\left(\alpha \bar{\varphi}_{0}-k^{2} \bar{\varphi}^{3 n}\right) \Delta t}{1+\left(\alpha+\beta k_{x}^{2}-k^{2}+k^{4}\right) \Delta t} .
$$

In the above numerical iteration, the double Fourier transform and its inverse of the order parameter $\varphi$ at each time step can be implemented by means of the efficient twodimensional fast Fourier transform (2D FFT) and its inverse (2D IFFT). The typical operations from time step $n$ to $n+1$ are the following:

(1) With $\varphi\left(x_{i}, y_{j}, t_{n}\right)$ and $\bar{\varphi}_{p q}\left(k, t_{n}\right)$ at time step $n$, evaluate $\varphi^{3}\left(x_{i}, y_{j}, t_{n}\right)$ at all spatial grids $\left(x_{i}, y_{j}\right)$.

(2) Perform 2D FFT to determine $\overline{\varphi_{p q}^{3}}\left(k, t_{n}\right)$.

(3) Evaluate relation (7) to determine $\bar{\varphi}_{p q}\left(k, t_{n+1}\right)$.

(4) Perform 2D IFFT to invert $\bar{\varphi}_{p q}\left(k, t_{n+1}\right)$ and then obtain $\varphi\left(x_{i}, y_{j}, t_{n+1}\right)$ at all spatial grids $\left(x_{i}, y_{j}\right)$ and time step $n+1$.

Due to use of the semi-implicit algorithm in the time domain and the Fourier-spectral scheme in spatial domain, the time steps can be selected as orders of those utilized in the conventional explicit Euler forward method. It has been validated that the present semi-implicit Fourier-spectral method is unconditionally stable in the sense that given a fixed set of physical parameters, there always exists a time threshold $\Delta t_{c}$, independent of the spatial grid size, such that for all $\Delta t$ $\leq \Delta t_{c}$, the above scheme is stable for all spatial grid sizes $[61,62]$. As a result, the present numerical scheme is capable of remarkably enhancing the computational efficiency and simultaneously improving the numerical stability, and therefore is much more efficient than the classic CDS.

\section{NUMERICAL RESULTS}

Within the framework of above computational scheme, one can find that three parameters (i.e., $\alpha, \beta$, and $\varphi_{0}$ ) in Eq. (5) dominate the entire phase separation in the BCP thin films. Among these, $\alpha$ and $\varphi_{0}$ are related to the BCP molecular structures and $\beta$ is linked to the combined effect of the external electric field and the BCP dielectric properties. Hereafter, we examine the effects of $\alpha, \beta$, and $\varphi_{0}$ on the self-assembly process in the BCP thin films within a $2 \mathrm{D}$ computational cell under periodic boundary conditions. The size of the computational cell in each simulation is fixed as $256 \times 256$. Sufficient large time step of 0.5 is fixed for the time integration of all cases in this study. The initial concentration of the order parameter $\varphi$ is assumed to have a Gaussian fluctuation of magnitude 0.002 around the average values designated in the following.

\section{A. Phase separation of symmetric BCP films in electric field}

Let us first consider the phase separation process in symmetric BCP thin films of varying $\alpha$ and $\beta$ (i.e., $\varphi_{0}=0$ ) in a lateral electric field along the $x$ axis. In the following simulations, the parameter $\alpha$ is selected as 0.1 and 0.2 , respectively, to show the effects of the BCP incompatibility and external electric field on the phase separation. For comparison, simulation of the phase separation free of electric field has also been performed. After 5000 iterations in each case, well-developed serpentine and lamellar nanostructures have formed in both neutral and electrified BCP thin films as shown in Figs. 1 and 2. The left column in Fig. 1 indicates the morphologies of order parameter $\varphi$ in the symmetric BCP film $(\alpha=0.1)$ at five sequential instants $(t=10,100$, 1000 , and 5000) during the phase separation process free of electric field $(\beta=0)$; while the right column in Fig. 1 shows those of the BCP film in the electric field of sufficient magnitude $(\beta=0.8)$. One can observe that the phase separation consists of phase nucleation and phase coarsening. By using the efficient semi-implicit Fourier-spectral scheme in this study, the time step has been selected very large in the iterations $(\Delta t=0.5)$. After 1000 iterations, the morphology of order parameter $\varphi$ for either neutral or electrified BCP films has tended to be stable though it continues evolving and coarsening afterwards. When subjected to lateral inplanar electric field, lamellar nanostructures are formed along the direction of the electric field as shown in the right column in Fig. 1. One can observe that phase dislocations exist in the lamellar nanostructures during the process of phase coarsening. The dislocations gradually disappear through climbing and molecular diffusion under the action of the electric driving force. Furthermore, compared to that of the left column in Fig. 1, the rate of electrified phase separation in the very beginning is lower than that free of the electric field ( $t=100$ for instance). This is because the molecular motion in a lateral electric field belongs to constrained motion. As a matter of fact, in the very beginning of phase separation, molecules in BCP films free of electric field have more degrees of freedom (DOFs) to move than those of electrified BCP films, i.e., higher rate of phase separation. However, after the beginning stage of phase nucleation, numerical results in Fig. 1 indicate that electric field has no noticeable effect on the rate of phase separation. In addition, Fig. 1 also shows that besides the formation of lamellar nanostructures, electric field has no significant effect on the extent of phase separation due to the characteristic of the global action exerted by the electric force on the molecular chains of the BCP films.

Furthermore, when subjected to weak lateral electric field, Fig. 2(a) shows the morphology of order parameter $\varphi$ of the above BCP thin films $(\alpha=0.1)$ after 5000 iterations. Compared to those in Fig. 1, numerous phase dislocations are detected in the simulation. As a result, the formation of lamellar nanostructures in BCP thin films can be accelerated through applying lateral electric field of high magnitude. For the purpose of examining the effect of molecular interaction on the phase separation process, Figs. 2(b)-2(d) show the morphologies of order parameter of the BCP films with $\alpha$ $=0.2$ after 5000 iterations. Figure 2(b) shows the results free of electric field; while Figs. 2(c) and 2(d) give these in a lateral electric field of $\beta=0.05$ and 0.1 , respectively. One can observe in Fig. 2(b) that for stronger monomer interaction $(\alpha=0.2)$, the extent of the corresponding phase separation is 


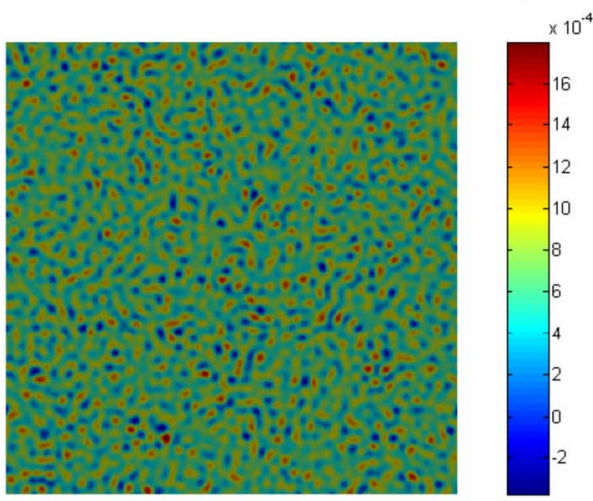

(a)

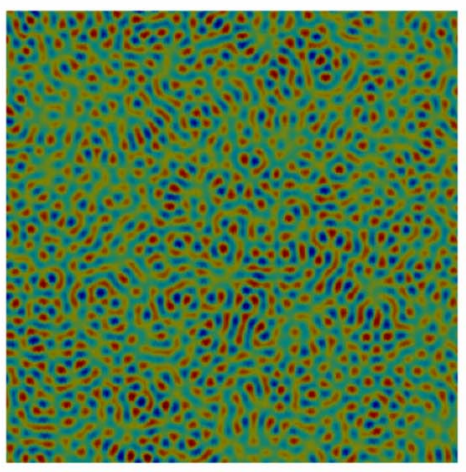

(b)

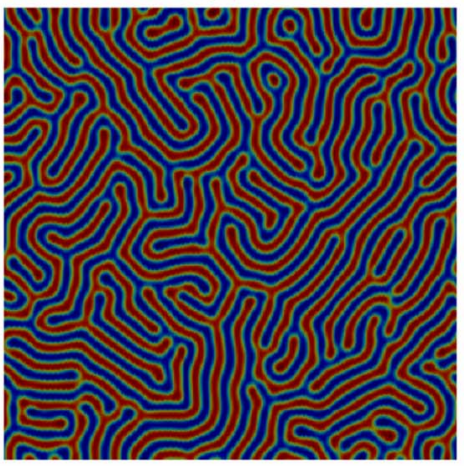

(c)

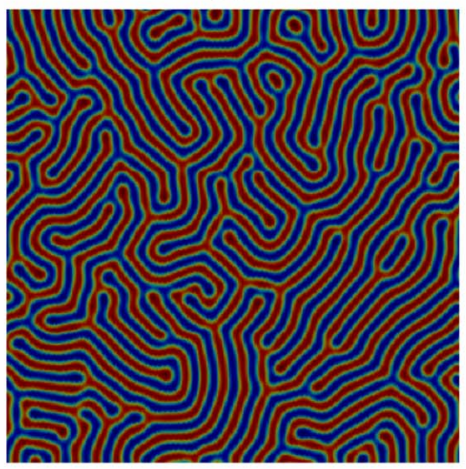

(d)
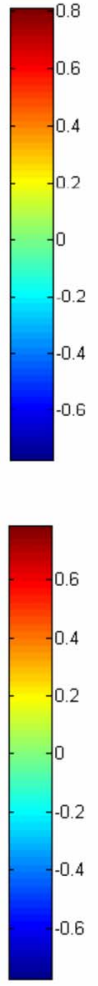

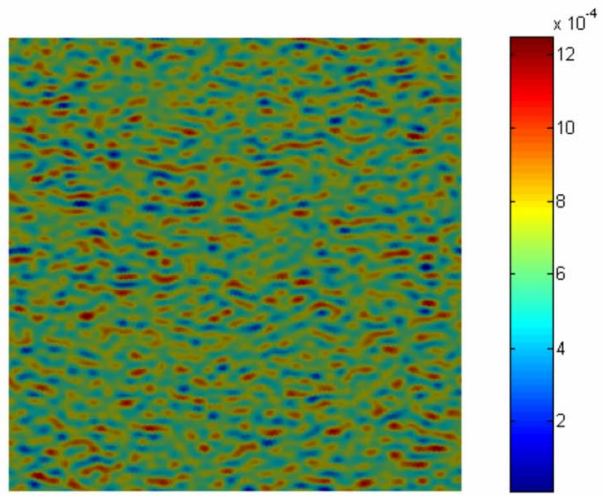

(e)

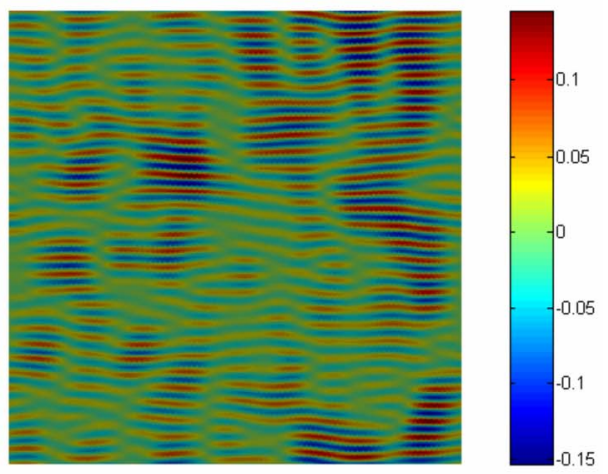

$(f)$

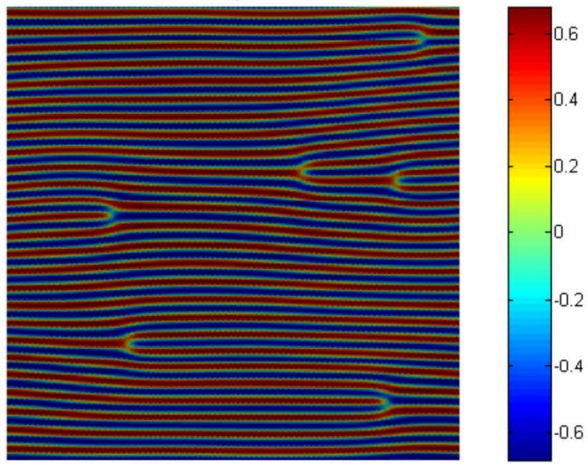

$(g)$

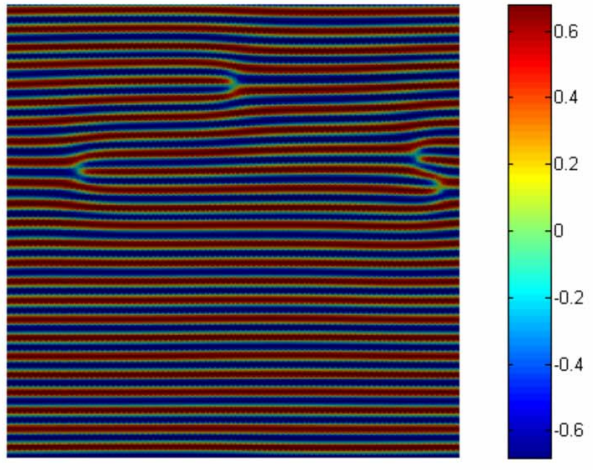

(h)

FIG. 1. (Color online) The morphology of order parameter $\varphi$ in symmetric BCP thin films $\left(\alpha=0.1\right.$ and $\left.\varphi_{0}=0\right)$. The left column are the numerical results of order parameter $\varphi$ at five sequential instants $(t=10,100,1000$, and 5000) in the phase separation process free of electric field, as indicated as (a), (b), (c), and (d), respectively. The right column are those obtained at the same five sequential instants $(t=10$, 100, 1000 , and 5000) in the case of lateral inplanar electric field $(\beta=0.8)$, as indicated as (e), (f), $(\mathrm{g})$, and (h), respectively. 


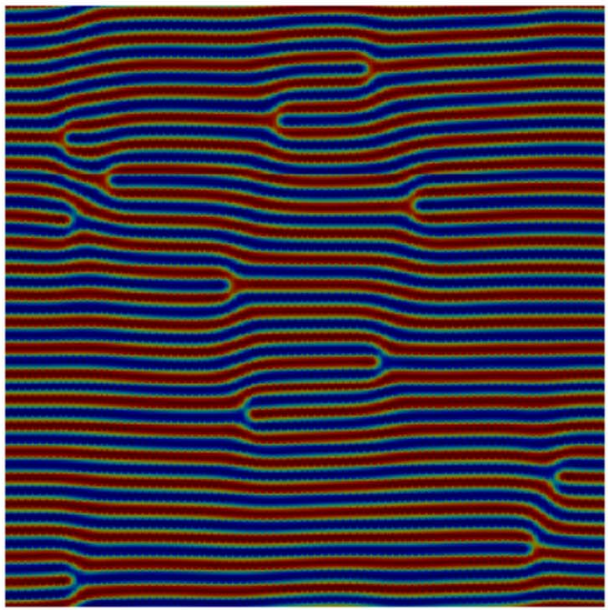

(a)

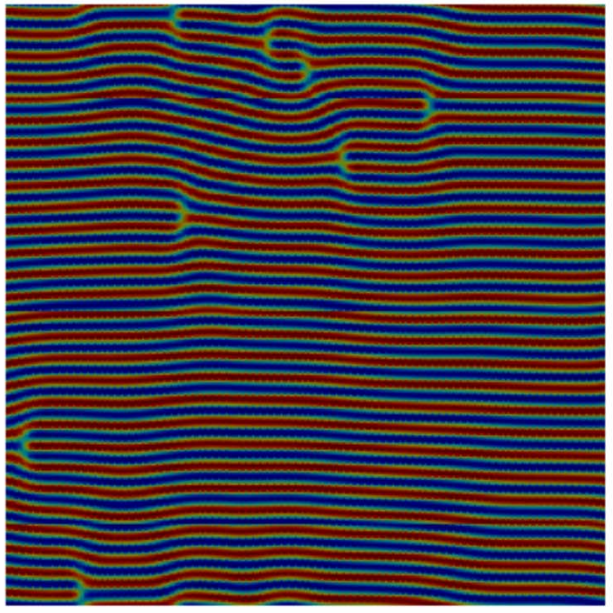

(c)
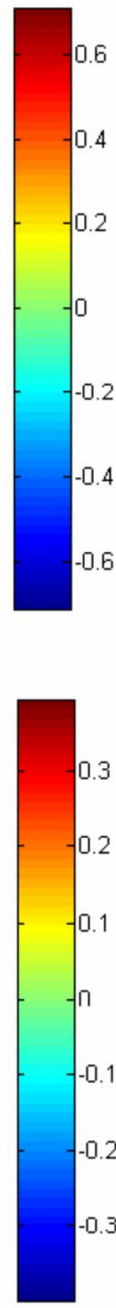

3
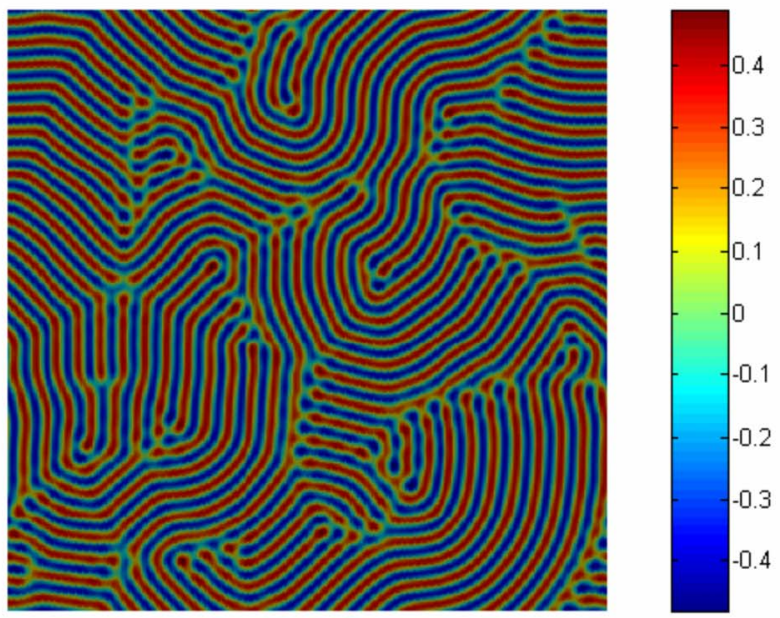

(b)

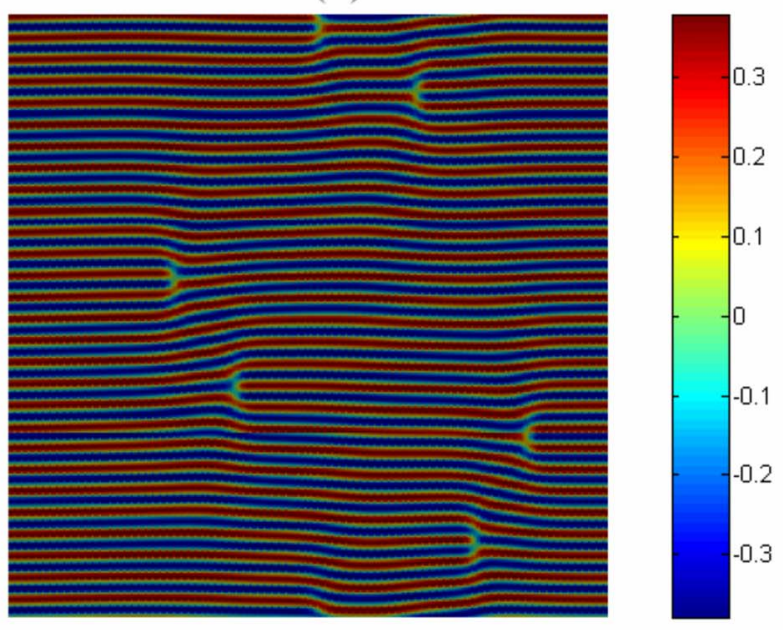

(d)

FIG. 2. (Color online) The morphology of order parameter $\varphi$ in symmetric BCP thin films $\left(\varphi_{0}=0\right)$ after 5000 iterations. (a) $\alpha=0.1$ and $\beta=0.1$ (in electric field), (b) $\alpha=0.2$ and $\beta=0$ (free of electric field), (c) $\alpha=0.2$ and $\beta=0.05$ (in electric field), and (d) $\alpha=0.2$ and $\beta=0.1$ (in electric field).

lower than that with weak monomer interaction such as $\alpha$ $=0.1$ (see Fig. 1). In the cases of relatively weak electric fields $(\beta=0.05$ and 0.1 , for instance), Figs. 2(c) and 2(d) indicate that a variety of phase dislocations exist due to the slow formation of lamellar nanostructures under weak electric driving forces.

\section{B. Phase separation of asymmetric BCP films in electric field}

Now let us consider the molecular self-assembly in asymmetric BCP thin films. Based on the above numerical scheme, Fig. 3 shows the morphologies of order parameter $\varphi$ in neutral and electrified asymmetric BCP thin films $(\alpha$ $=0.05$ and $\left.\varphi_{0}=0.35\right)$ at five sequential instants $t=10,100$, 1000, and 5000, respectively. From Fig. 3, one can observe that well-defined morphologies of random nanodots and lamellar nanostructures have formed after 5000 iterations. The left column are these free of electric field, while the right column are those in lateral electric field $(\beta=1.8)$. Similar to that of symmetric BCPs as discussed in Sec. III A, the phase separation process in this case also consists of phase nucleation and phase coarsening to reduce the global free energy of the BCP system. For asymmetric BCP films free of electric field, coarsening of the random nanodots accompanies with the annihilation of unstable nanodots (usually in relatively smaller size) through molecular diffusion. When subjected to relatively high lateral electric field, the right column of Fig. 3 shows that lamellar nanostructures begin to form in the very beginning of the process. In this case, it can be found that with the aid of the electric driving force, phase dislocations nucleate and then climb to reduce the global free energy. Furthermore, due to the introduction of electric field of high magnitude, sphere-to-cylinder domain transition happened in the very beginning of the process. In order to examine this transition, we also examine the phase separation process in the same BCP thin film in weak electric field $(\beta$ $=0.2$ ). After 5000 iterations, the morphology of order parameter in Fig. 4(a) shows a variety of intermediate phase struc- 


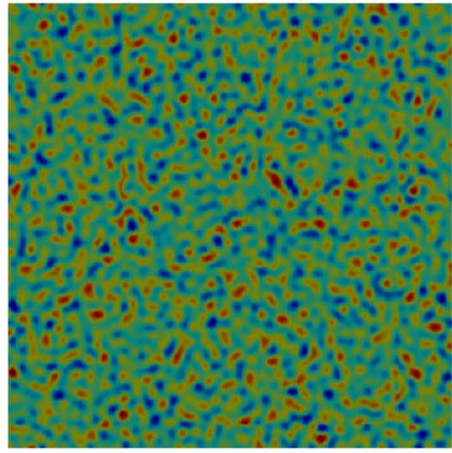

(a)

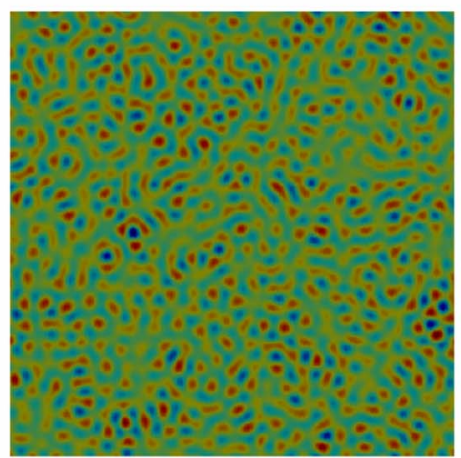

(b)

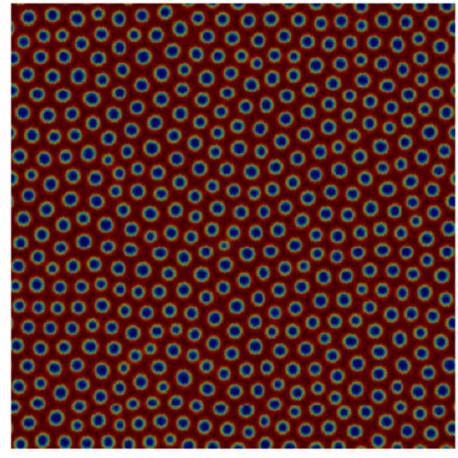

(c)

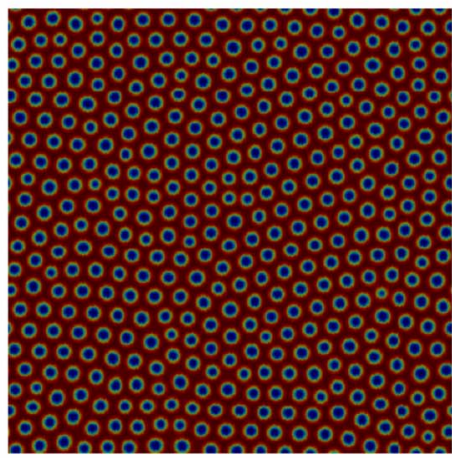

(d)
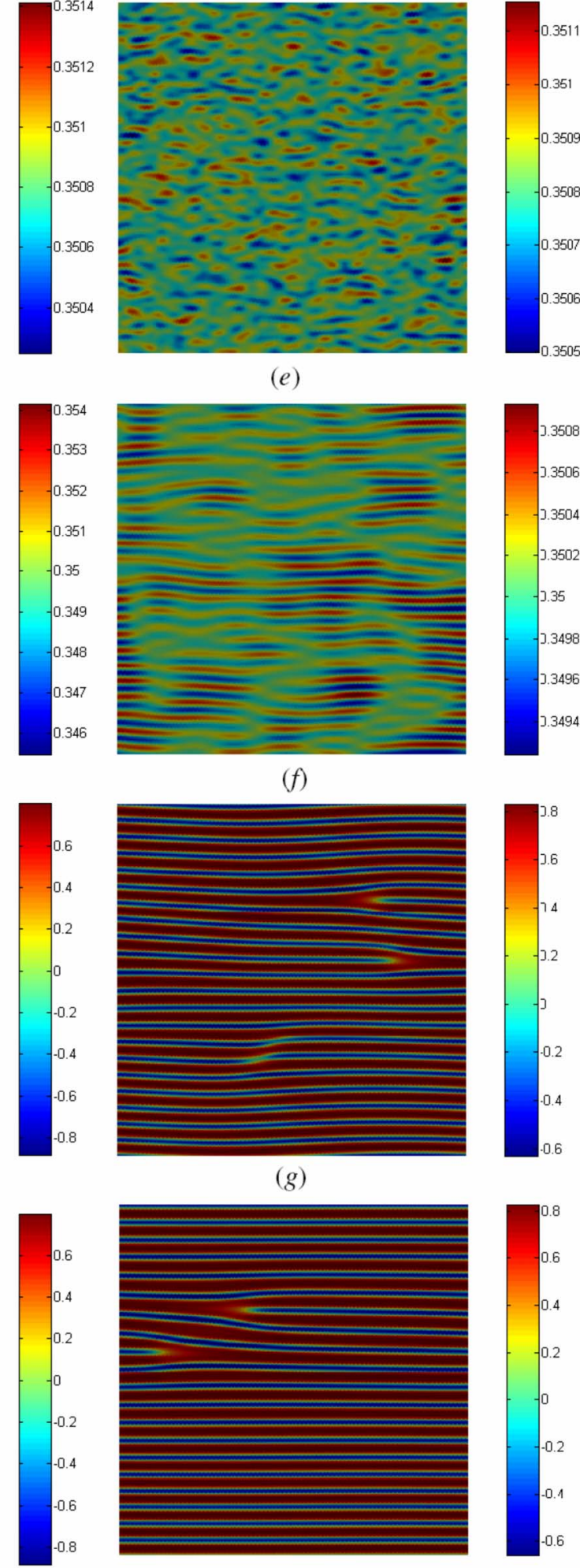

(h)

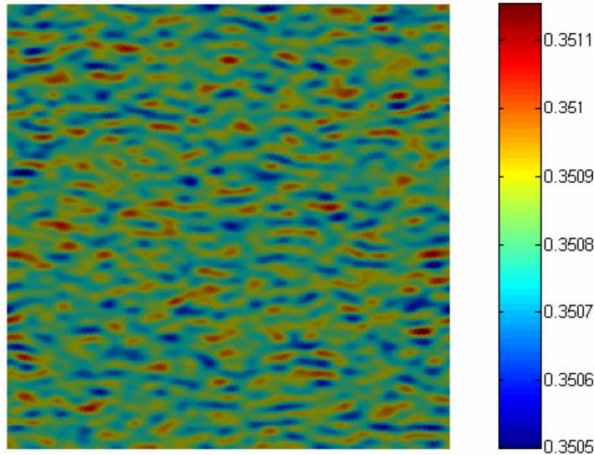

(e)

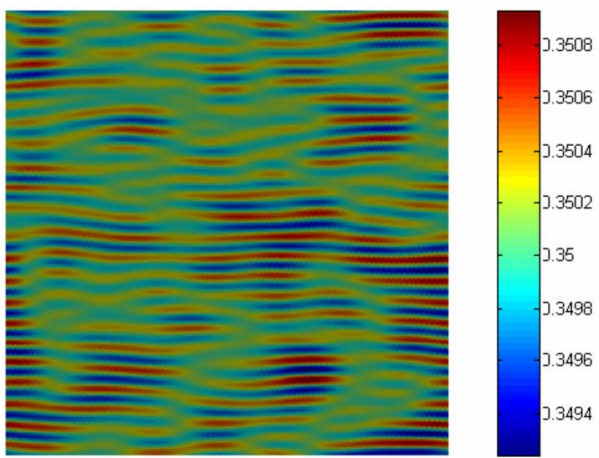

$(f)$

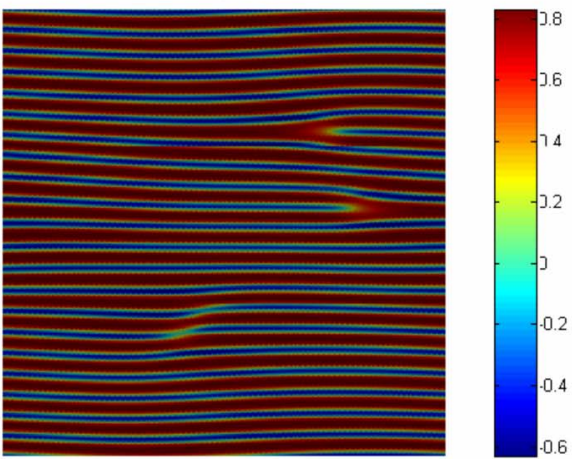

$(g)$

FIG. 3. (Color online) The morphology of order parameter $\varphi$ in asymmetric BCP thin films $\left(\alpha=0.05\right.$ and $\left.\varphi_{0}=0.35\right)$. The left column are the numerical results of order parameter $\varphi$ at five sequential instants $(t=10,100,1000$, and 5000) in the phase separation process free of electric field, as indicated as (a), (b), (c), and (d), respectively. The right column are those results obtained at the same five sequential instants $(t=10,100,1000$, and 5000) in the case of lateral inplanar electric field $(\beta=1.8)$, as indicated as (e), (f), (g), and (h), respectively. 


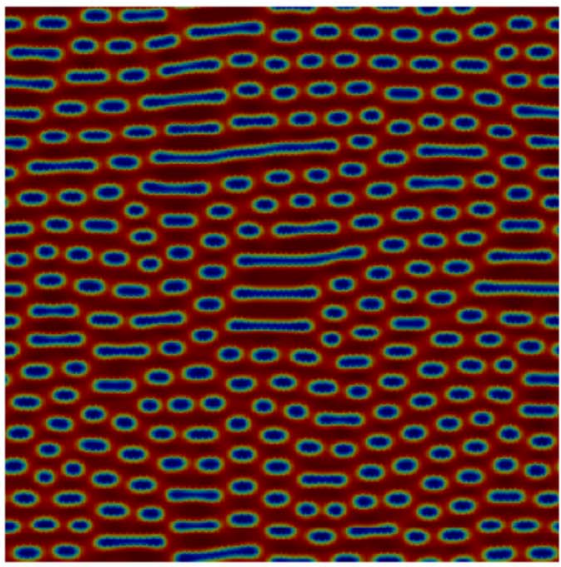

(a)

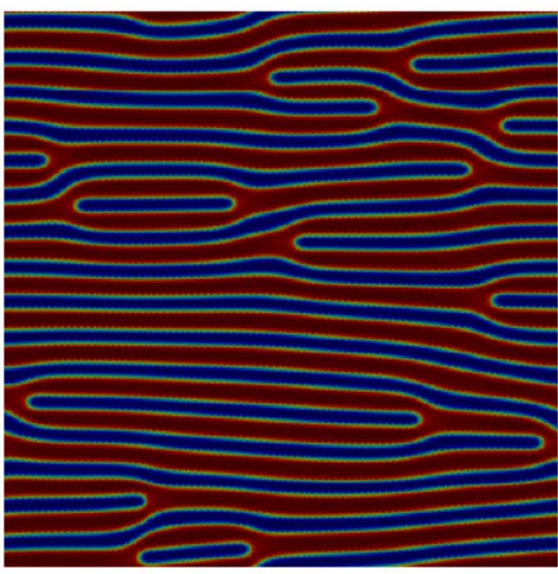

(c)
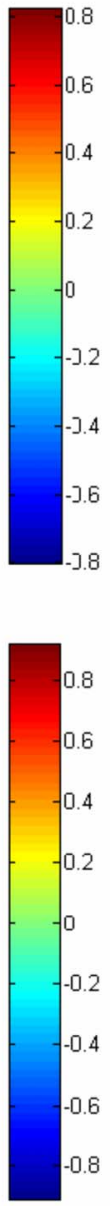
0.8
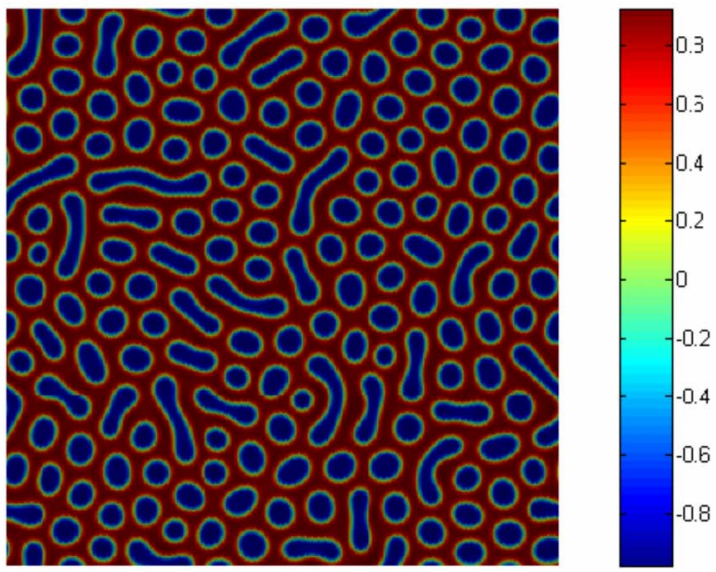

(b)

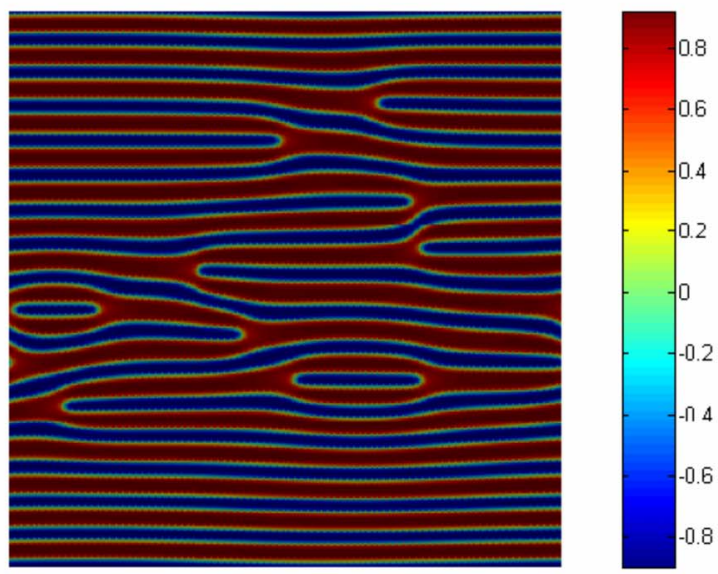

(d)

FIG. 4. (Color online) The morphology of order parameter $\varphi$ in asymmetric BCP thin films after 5000 iteration. (a) $\alpha=0.05, \varphi_{0}=0.35$, and $\beta=0.2$ (in electric field), (b) $\alpha=0.02, \varphi_{0}=0.15$, and $\beta=0$ (free of electric field), (c) $\alpha=0.02, \varphi_{0}=0.15$, and $\beta=0.2$ (in electric field), and (d) $\alpha=0.02, \varphi_{0}=0.15$, and $\beta=0.4$ (in electric field).

tures including spheres, ellipsoids, and cylinders, similar to those observed in recent experiments [32,53].

In addition, we also investigate the phase separation process in neutral $(\beta=0)$ and electrified $(\beta=0.2$ and 0.4$)$ asymmetric BCP thin films $\left(\alpha=0.02\right.$ and $\left.\varphi_{0}=0.15\right)$. In the case free of electric field, after 5000 iterations, the morphology of order parameter includes random nanodots and nanoislands as shown in Fig. 4(b); and Figs. 4(c) and 4(d) show the intermediate lamellar nanostructures of the BCP films in relatively weak electric fields with $\beta=0.2$ and 0.4 , respectively. In the latter cases, the lamellar nanostructures are still evolving. This formation process can be accelerated by increasing the magnitude of electric field.

\section{Domain size evolution}

In the above, the morphology of order parameter in $\mathrm{BCP}$ thin films has been determined through detailed phaseseparation modeling. In this section, we further consider the evolution of domain size by introducing the transient average domain size $R_{y}(t)$ along the $y$ direction, which is defined by the inverse of the transient average wave numbers $\left\langle k_{y}(t)\right\rangle$ $[63,67-69]$ as

$$
R_{y}(t)=2 \pi /\left\langle k_{y}(t)\right\rangle
$$

where

$$
\left\langle k_{y}(t)\right\rangle=\frac{\sum_{k_{x}} \sum_{k_{y}} k_{y} S\left(k_{x}, k_{y}, t\right)}{\sum_{k_{x}} \sum_{k_{y}} S\left(k_{x}, k_{y}, t\right)} .
$$

In relations (8) and (9) $k_{y}$ is the variable in Fourier domain, and $S\left(k_{x}, k_{y}, t\right)$ is the element of the structure factor matrix defined by [67-69]

$$
S\left(k_{x}, k_{y}, t\right)=\frac{1}{N} \bar{\varphi}\left(k_{x}, k_{y}, t\right) \bar{\varphi}\left(-k_{x},-k_{y}, t\right)-\langle\varphi\rangle^{2} I\left(k_{x}, k_{y}\right),
$$

where $N=L^{2}$ is the total number of grids within the computational cell; $\langle\varphi\rangle$ is the average order parameter; and $\bar{\varphi}\left(k_{x}, k_{y}, t\right)$ and $I\left(k_{x}, k_{y}\right)$ are the coefficients of 2D FFT of 


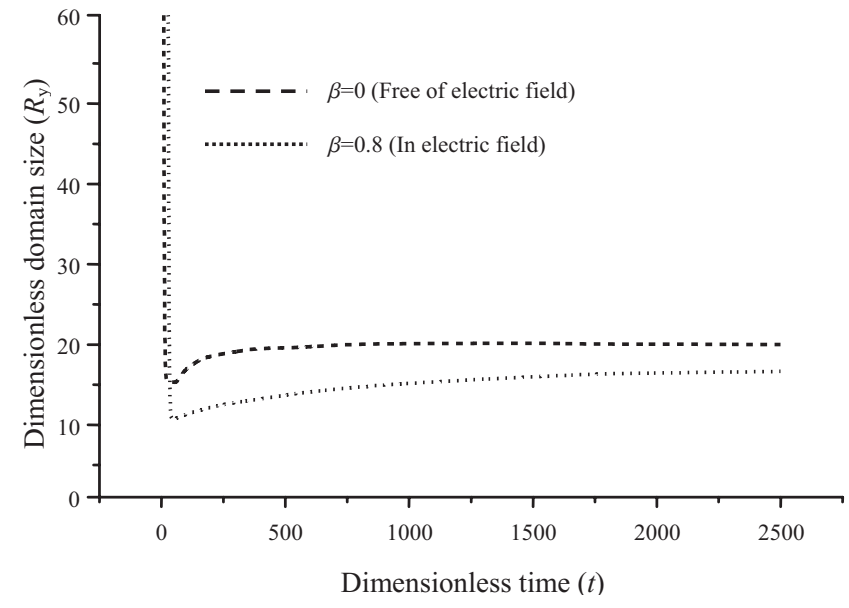

FIG. 5. Variation of the domain size $R_{y}(t)$ vs the time of the symmetric BCP thin films $\left(\alpha=0.1\right.$ and $\left.\varphi_{0}=0\right)$ during the molecular self-assembly process.

$\varphi(x, y, t)$ and the unit in the computational cell, respectively. With definition (8), Figs. 5 and 6 show the evolution of domain size of the BCP thin films in above simulations. It can be observed that the domain size along $y$ direction $R_{y}(t)$ in each case decreases abruptly in the very beginning of phase separation (i.e., the phase nucleation stage), and then tends to constant through phase coarsening in both the symmetric and asymmetric BCP films. In the case of symmetric BCP thin films $\left(\alpha=0.1\right.$ and $\left.\varphi_{0}=0\right)$ free of electric field, the stable $R_{y}(t)$ is close to 20; while in lateral electric field $(\beta=0.8)$, the corresponding stable $R_{y}(t)$ is reduced to around 17. In the case of asymmetric BCP thin film $\left(\alpha=0.05\right.$ and $\left.\varphi_{0}=0.35\right)$ free of electric field, the stable $R_{y}(t)$ is close to 17.5 ; when introducing a lateral electric field $(\beta=1.8)$, the stable $R_{y}(t)$ is reduced to $\sim 13.5$. This is because the lateral electric field is able to "organize" the random nanostructures, usually formed free of electric field, into highly ordered lamellar nanostructures along the direction of the inplanar electric field as shown in Figs. 1-4. Such "electric stretching" decreases the domain size of the random nanostructures in BCP thin films perpendicular to the direction of the inplanar electric field. Thus, one can draw the conclusion that the adoption of lateral electric field can decrease the transverse domain size $R_{y}(t)$ for both symmetric and asymmetric BCP thin films.

\section{CONCLUDING REMARKS}

In this study, we have examined the molecular selfassembly in both symmetric and asymmetric BCP thin films by solving the resulting $\mathrm{CH}$ equation numerically. Detailed morphology of order parameter of the BCP thin films and the evolution of domain size have been examined. It shows that

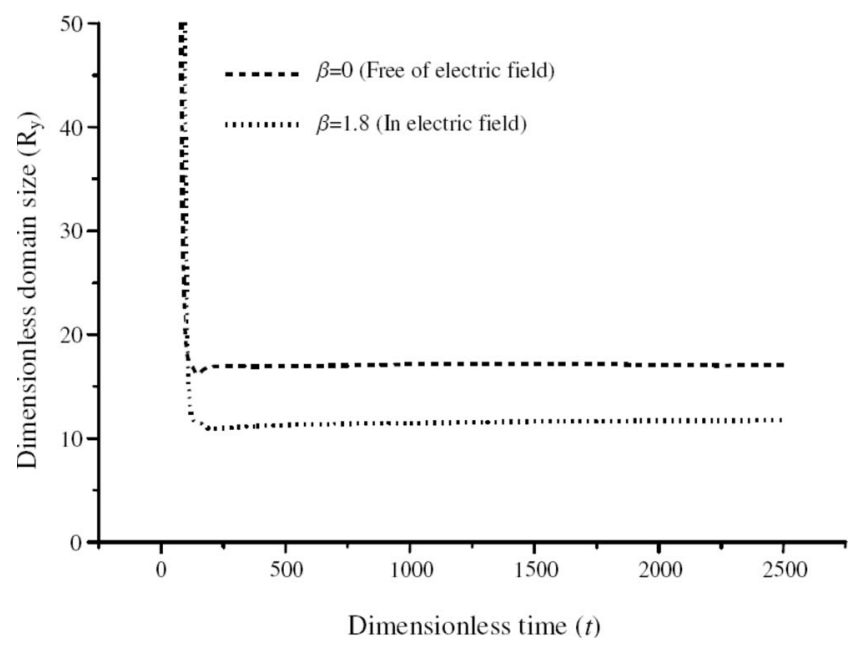

FIG. 6. Variation of the domain size $R_{y}(t)$ vs the time of the asymmetric BCP thin films $\left(\alpha=0.05\right.$ and $\left.\varphi_{0}=0.35\right)$ during the molecular self-assembly process.

the semi-implicit Fourier-spectral algorithm adopted in this study can obviously enhance the computational efficiency and numerical stability.

Numerical results indicate that lateral electric field can be employed to form ordered lamellar nanostructures in both symmetric and asymmetric BCPs. The rate of phase separation in the BCP thin films depends upon both the type of molecular chain structures and the magnitude of electric field. The entire process of forming lamellar nanostructures consists of phase nucleation and phase coarsening through climbing of phase dislocations and molecular diffusion. The present study also indicates that external electric field has no noticeable effect on the extent of phase separation. For asymmetric BCP thin films in electric field of sufficient magnitude, sphere-to-cylinder transition happened during the formation of lamellar nanostructures. In addition, the domain size decreases accordingly with the formation of ordered lamellar nanostructures due to the "electric stretching" effect.

In practice, lateral electric field can be introduced conveniently, thus electrically guided molecular self-assembly in $\mathrm{BCP}$ thin films can be utilized efficiently in manufacturing highly ordered nanostructures and nanodevices. Moreover, the numerical method presented in this study can be used for intelligent BCP-based nanomanufacturing for targeted nanostructures through optimizing the applied electric field, and it can also be used for the analysis and prediction of the process of nanostructure formation in BCP thin films.

\section{ACKNOWLEDGMENT}

Partial support of this work by the NSF/AFOSR/ARO/ ARL is greatly appreciated. 
[1] I. W. Hamley, The Physics of Block Copolymer (Oxford University Press, Oxford, 1998).

[2] M. J. Fasolka and A. M. Mayes, Annu. Rev. Mater. Res. 31, 323 (2001).

[3] N. Hadjichristidis, S. Pispas, and G. Floudas, Block Copolymer-Synthetic Strategies, Physical Properties, and Application (Wiley, New York, 2003).

[4] I. Norde, Surface and Interfacial Aspects of Biomedical Applications (Plenum, New York, 1995).

[5] V. Castelletto and I. W. Hamley, Curr. Opin. Solid State Mater. Sci. 8, 426 (2004).

[6] F. S. Bates, MRS Bull. 30, 525 (2005).

[7] M. V. Tirrell and A. Katz, MRS Bull. 30, 700 (2005)

[8] M. D. Ward, MRS Bull. 30, 705 (2005).

[9] T. Bein, MRS Bull. 30, 713 (2005).

[10] J. Yoon, W. Lee, and E. L. Thomas, MRS Bull. 30, 721 (2005).

[11] H. Colfen and S.-H. Yu, MRS Bull. 30, 727 (2005).

[12] M. Boncheva and G. M. Whitesides, MRS Bull. 30, 736 (2005).

[13] C. J. Hawker and T. P. Russell, MRS Bull. 30, 952 (2005).

[14] L. Rockford, Y. Liu, P. Mansky, T. P. Russell, M. Yoon, and S. G. J. Mochrie, Phys. Rev. Lett. 82, 2602 (1999).

[15] W. A. Lopes and H. M. Jaeger, Nature (London) 414, 735 (2001).

[16] S. O. Kim, H. H. Solak, M. P. Stoykovich, N. J. Ferrier, J. J. dePablo, and P. F. Nealey, Nature (London) 424, 411 (2003).

[17] N. Kumar and J. I. Hahm, Langmuir 21, 6652 (2005).

[18] M. P. Stoykovich, M. Muller, S. O. Kim, H. H. Solak, E. W. Edwards, J. J. de Pablo, and P. F. Nealey, Science 308, 1442 (2005).

[19] C. Harrison, D. H. Adamson, Z. D. Cheng, J. M. Sebastian, S. Sethuraman, D. A. Huse, R. A. Register, and P. M. Chaikin, Science 290, 1558 (2000).

[20] E. W. Edwards, M. P. Stoykovich, M. Muller, H. H. Solak, J. J. De Pablo, and P. F. Nealey, J. Polym. Sci., Part B: Polym. Phys. 43, 3444 (2005).

[21] L. Rockford, S. G. J. Mochrie, and T. P. Russell, Macromolecules 34, 1487 (2001).

[22] R. A. Segalman, A. Hexemer, R. C. Hayward, and E. J. Kramer, Macromolecules 36, 3272 (2003).

[23] J. Y. Cheng, C. A. Ross, E. L. Thomas, H. I. Smith, and G. J. Vancso, Adv. Mater. (Weinheim, Ger.) 15, 1599 (2003).

[24] J. Y. Cheng, A. M. Mayes, and C. A. Ross, Nat. Mater. 3, 823 (2004).

[25] K. C. Daoulas and M. Müller, Phys. Rev. Lett. 96, 036104 (2006).

[26] T. L. Morkved, M. Lu, A. M. Urbas, E. E. Ehrichs, H. M. Jaeger, P. Mansky, and T. P. Russell, Science 273, 931 (1996).

[27] P. Mansky, J. DeRouchey, T. P. Russell, J. Mays, M. Pitsikalis, T. Morkved, and H. Jaeger, Macromolecules 31, 4399 (1998).

[28] T. L. Morkved, W. A. Lopes, J. Hahn, S. J. Sibener, and H. M. Jaeger, Polymer 39, 3871 (1998).

[29] A. Boker, H. Elbs, H. Hansel, A. Knoll, S. Ludwigs, H. Zettl, V. Urban, V. Abetz, A. H. E. Muller, and G. Krausch, Phys. Rev. Lett. 89, 135502 (2002).

[30] S. Elhadj, J. W. Woody, V. S. Niu, and R. F. Saraf, Appl. Phys. Lett. 82, 871 (2003).
[31] T. Xu, Y. Q. Zhu, S. P. Gido, and T. P. Russell, Macromolecules 37, 2625 (2004).

[32] T. Xu, A. V. Zvelindovsky, G. J. A. Sevink, O. Gang, B. Ocko, Y. Q. Zhu, S. P. Gido, and T. P. Russell, Macromolecules 37, 6980 (2004).

[33] J. Y. Wang, T. Xu, J. M. Leiston-Belanger, S. Gupta, and T. P. Russell, Phys. Rev. Lett. 96, 128301 (2006).

[34] V. Olszowka, M. Hund, V. Kuntermann, S. Scherdel, L. Tsarkova, A. Boker, and G. Krausch, Soft Matter 2, 1089 (2006).

[35] D. E. Angelescu, J. H. Waller, D. H. Adamson, P. Deshpande, S. Y. Chou, R. A. Register, and P. M. Chaikin, Adv. Mater. (Weinheim, Ger.) 16, 1736 (2004).

[36] D. E. Angelescu, J. H. Waller, R. A. Register, and P. M. Chaikin, Adv. Mater. (Weinheim, Ger.) 17, 1878 (2005).

[37] G. Arya, J. Rottler, A. Z. Panagiotopoulos, D. J. Srolovitz, and P. M. Chaikin, Langmuir 21, 11518 (2005).

[38] D. B. Scott, A. J. Waddon, Y. G. Lin, F. E. Karasz, and H. H. Winter, Macromolecules 25, 4175 (1992).

[39] H. H. Winter, D. B. Scott, W. Gronski, S. Okamoto, and T. Hashimoto, Macromolecules 26, 7236 (1993).

[40] Z. R. Chen and J. A. Kornfield, Polymer 39, 4679 (1998).

[41] S. Kitade, N. Ochiai, Y. Takahashi, I. Noda, Y. Matsushita, A. Karim, A. I. Nakatani, H. D. Kim, and C. C. Han, Macromolecules 31, 8083 (1998).

[42] D. L. Polis, S. D. Smith, N. J. Terrill, A. J. Ryan, D. C. Morse, and K. I. Winey, Macromolecules 32, 4668 (1999).

[43] G. Arya, J. Rottler, A. Z. Panagiotopoulos, D. J. Srolovitz, and P. M. Chaikin, Langmuir 21, 11518 (2005).

[44] I. Rychkov, Macromol. Theory Simul. 14, 207 (2005).

[45] M. W. Wu, R. A. Register, and P. M. Chaikin, Phys. Rev. E 74, 040801 (2006).

[46] Y. Tsori and D. Andelman, Macromolecules 35, 5161 (2002).

[47] Y. Tsori, F. Tournilhac, D. Andelman, and L. Leibler, Phys. Rev. Lett. 90, 145504 (2003).

[48] C. Y. Lin, M. Schick, and D. Andelman, Macromolecules 38, 5766 (2005).

[49] C. Y. Lin and M. Schick, J. Chem. Phys. 125, 034902 (2006).

[50] M. W. Matsen, Phys. Rev. Lett. 95, 258302 (2005).

[51] M. W. Matsen, Soft Matter 2, 1048 (2006).

[52] M. W. Matsen, J. Chem. Phys. 124, 074906 (2006).

[53] A. V. Kyrylyuk, G. J. A. Sevink, A. V. Zvelindovsky, and J. G. E. M. Fraaije, Macromol. Theory Simul. 12, 508 (2003).

[54] M. Pinna, A. V. Zvelindovsky, S. Todd, and G. GoldbeckWood, J. Chem. Phys. 125, 154905 (2006).

[55] A. V. Kyrylyuk and J. G. E. M. Fraaije, J. Chem. Phys. 125, 164716 (2006)

[56] S. A. Brazovskii, Sov. Phys. JETP 41, 85 (1975).

[57] L. Leibler, Macromolecules 13, 1602 (1980).

[58] T. Ohta and K. Kawasaki, Macromolecules 19, 2621 (1986).

[59] G. H. Fredrickson, Macromolecules 20, 2535 (1987).

[60] G. H. Fredrickson and E. Helfand, J. Chem. Phys. 87, 697 (1987).

[61] L. Q. Chen and J. Shen, Comput. Phys. Commun. 108, 147 (1998).

[62] J. Z. Zhu, L. Q. Chen, J. Shen, and V. Tikare, Phys. Rev. E 60, 3564 (1999).

[63] X. F. Wu and Y. A. Dzenis, J. Chem. Phys. 125, 174707 (2006). 
[64] P. C. Hohenberg and B. I. Halperin, Rev. Mod. Phys. 49, 435 (1977).

[65] K. Amundson, E. Helfand, X. Quan, and S. D. Smith, Macromolecules 26, 2698 (1993).
[66] A. Onuki and J. Fukuda, Macromolecules 28, 8788 (1995).

[67] Y. Oono and S. Puri, Phys. Rev. A 38, 434 (1988).

[68] S. Puri and Y. Oono, Phys. Rev. A 38, 1542 (1988).

[69] A. Shinozaki and Y. Oono, Phys. Rev. A 45, R2161 (1992). 